\title{
NIVEL DE FLEXIBILIDAD DE DEPORTISTAS EN FORMACIÓN A TRAVÉS DEL TEST DE SIT AND REACH, TOCANCIPÁ, CUNDINAMARCA
}

\author{
LEVEL OF FLEXIBILITY OF ATHLETES IN TRAINING THROUGH THE TEST OF SIT AND REACH, \\ TOCANCIPÁ, CUNDINAMARCA.
}

Jenny Paola Peraza Gómez

Estudiante de Ciencias del Deporte de la Universidad de Ciencias Aplicadas y Ambientales

E-mail: paoperazag95@hotmail.com

\section{Angie Lizeth Castañeda Casasbuenas}

Estudiante de Ciencias del Deporte de la Universidad de Ciencias Aplicadas y Ambientales

E-mail: angie_stich@hotmail.es

\section{Diana Marcela Zapata Torres}

Magister Universidad Nacional de Colombia sede Bogotá, maestría fisioterapia deportiva y actividad física.

E-mail: diazapatat@udca.edu.co

\section{Danny Wilson Sanjuanelo Corredor}

Ingeniero Agrónomo.

Especialista en Docencia para la educación superior.

Departamento de Ciencias Exactas y

Naturales.Facultad de Ciencias, U.D.C.A

E-mail: dsanjuanelo@udca.edu.co

\section{RESUMEN}

El estudio determinó el nivel de flexibilidad, a través del test Sit and Reach en los niños y niñas de los diferentes deportes en formación del Instituto Municipal de Recreación y de Deporte de Tocancipá. Estudio de tipo descriptivo con enfoque cuantitativo, con un rango de edad de la población entre los 8 y 17 años, con la participación de 334 niñas y niños. El mejor registro en el test Sit and Reach, se presentó en niñas a los 14 años con un valor de $(25,9 \pm 6,4 \mathrm{~cm})$, en niños de $(19,3 \pm 7,6 \mathrm{~cm})$, para la variable deporte en la que se midieron 13 deportes, capoeira en niñas obtuvo un valor de $(29,5 \pm 3,8 \mathrm{~cm})$, en niños $(24,5 \pm 7,1 \mathrm{~cm})$, siendo capoeira el deporte con mejor resultado en ambos sexos, de acuerdo al percentil 90,87 niños y niñas se encuentran por encima del valor, mientras 65 niños y niñas están por debajo del percentil 20, confirmando la hipótesis alternativa, (p: 0,0087).

Palabras clave: Valoración en deportista en formación, niños, flexibilidad, Sit and Reach.

\section{ABSTRACT}

The study determined the level of flexibility through the Sit and Reach test in children of different sports in formation of the Municipal Institute of recreation and sport of Tocancipá. Descriptive study with quantitative approach, with an age range of the population between 8 and 17 years old, with the participation of 334 children. The best record in the Sit and Reach test, arose in girls at age 14 with a value of $(25,9 \pm 6,4 \mathrm{~cm})$, in children's $(19,3 \pm 7,6 \mathrm{~cm})$, a value is obtained for the variable sport in which 
measured 13 sports, capoeira in girls of (29, $5 \pm 3,8 \mathrm{~cm})$, in children $(24,5 \pm 7,1 \mathrm{~cm})$, being capoeira sports with best result in both sexes, according to the percentile 90,87 children are above the value, while 65 children and girls are below the 20 percentile, confirming the alternative hypothesis ( $p$ : 0,0087).

Key words: Assessment in training athlete, children, flexibility, Sit and Reach

\section{INTRODUCCIÓN}

La presente investigación se realizó en el Municipio de Tocancipá, Cundinamarca, desarrollando una medición en un macro proyectó usando las siguientes variables, (peso, talla, IMC zscore, \% grasa, circunferencia de cintura, fuerza prensil, flexibilidad, salto largo, velocidad y resistencia), seleccionando para el desarrollo del presente trabajo la variable flexibilidad (Test Sit and Reach), que es denominada como: "la capacidad de mover una articulación a lo largo de todo el rango movimiento, asociado a la amplitud de movimiento puede dividirse en dos tipos: movilidad activa (acción de los grupos musculares sin ninguna resistencia) y la pasiva (requiere ayuda externa), es un componente de la aptitud física relacionado con la salud y con el rendimiento" American (College of Sports Medicine, 2010), el abordaje de esta variable permite identificar la capacidad del sistema musculo esquelético para permitir el desarrollo de movimientos esenciales en el desarrollo formativo de los niños y niñas.

Como variables condicionantes en el desarrollo de test físicos, se prioriza en la edad $y$ sexo, que van a tener un comportamiento diferencial acorde a cada etapa de crecimiento, involucrando dos factores importantes al momento de realizar un análisis de la información recolectada, en este caso en particular la población objeto de estudio son niños y niñas que participan del programa de deporte formativo del municipio de Tocancipá, Cundinamarca, que a nivel deportivo es uno de los departamentos que se ha destacado por su gran aporte en el área, desde su formación de semilleros en diversas modalidades deportivas, destacándose a nivel nacional, este direccionamiento de apoyar la región de Cundinamarca se centra desde la formación de la zona sabana centro, en el que se encuentra el Municipio de Tocancipá.

La flexibilidad es una habilidad fundamental que se debe mantener a lo largo de la vida, que genera una necesidad de entrenamiento habitual, para evitar un declive del valor ponderal de esta cualidad, por tanto, se hace necesario realizar ejercicios destinados a una menor pérdida, preferentemente, de flexibilidad estática (Blanco, A., 2015).

Adicionalmente, es importante resaltar las características que se destacan de la flexibilidad entre los 10 a 13 años de vida, donde producen diferencias considerables de acuerdo a la maduración sexual, teniendo en cuenta que: "las mujeres suelen ser más flexibles que los hombres por las diferencias hormonales que presentan. La mayor producción de estrógenos en las mujeres causa una disminución de la viscosidad de los tejidos" (Hernández, D., 2006). 


\section{METODOLOGÍA}

Se considera como punto de partida en los procesos deportivos, que la evaluación de la condición física y el desarrollo de protocolos establecidos, pueden generar un estímulo de motivación en los niños y niñas, adicionalmente, conocer el nivel de condición física, verificar progresos, identificar factores de riesgo, diseñar programas de actividad física, promover la salud o fomentar el desarrollo deportivo (Martín, S., 2016), para generar un crecimiento a nivel personal.

Este proceso se realiza mediante el desarrollo de procesos de medición, resaltándose en este caso en particular la importancia de ejecutar la evaluación para la cualidad física de flexibilidad a través del test Sit and Reach, que reporta una validez en el instrumento de medida, teniendo en cuenta, que se define como el grado de precisión con el que el test mide aquello que se ha propuesto medir, es decir, el grado con el que cumple su objetivo, al igual que la fiabilidad, la validez del instrumento puede verse comprometida por las características individuales de la población que se usaron en la investigación (Ayala, F., 2012).

El presente trabajo de investigación permite determinar: cuáles son los valores en el test de Sit and Reach, en los niños y niñas pertenecientes al Instituto Municipal de Recreación y de Deporte de Tocancipá, indicando el nivel de flexibilidad en la musculatura isquiotibial. Por otra parte, este trabajo investigativo servirá de apoyo para otros investigadores que quieran evaluar la condición física a nivel poblacional en la cualidad física de flexibilidad y sea un referente para futuras investigaciones en el ámbito académico, generando así, un impacto social en la población, lo que permita mejorar el rendimiento deportivo desde tempranas edades.

A continuación, se presenta una descripción de los artículos relacionados con la flexibilidad. En cuanto a la flexibilidad, es necesario resaltar que esta capacidad es fundamental en procesos de formación, así lo mencionan en sus investigaciones, (Allen, W., Jackson, A.,

Baker, A., 1986), en un estudio con niñas de 13 a 15 años, dedicado a la validez del "sentarse y alcanzar" para medir la flexibilidad de la parte inferior de la espalda y de los tendones de la parte posterior de la rodilla, llegan a la conclusión que tiene una validez moderada, cuando se use sólo como una valoración de flexibilidad de tendones, de la parte posterior de la rodilla.

Así mismo, encontramos que (Docherty, D. y Bell, R., 1985) tomaron niños y niñas de $6,9,12$ y 15 años, para hallar la flexibilidad con medidas lineales (Sit and Reach, elevación del hombro y muñeca, extensión del tronco y cuello), medidas angulares con el flexómetro Leigthon (flexión / extensión de hombro, cadera y tronco), en las que se estableció una relación de medidas antropométricas y concluyen que las niñas eran más flexibles que los niños en todas las edades, para los niños la flexibilidad declina con la edad, tendencia particularmente cierta para la flexión/extensión de hombro, flexión / extensión de tronco, sentarse y alcanzar, elevación del hombro y muñeca, extensión del tronco y cuello. Es explicable por las diferencias en la morfología del esqueleto entre los dos sexos.

Por otra parte, Sainz, P. (2009) en la investigación, tuvo como objetivo analizar el efecto de un programa de estiramientos de la musculatura isquiosural en escolares de Enseñanza Secundaria Obligatoria. Un total de 50 escolares pertenecientes a 2 cursos de 
$2^{\circ}$ de la ESO (peso: $55,18 \pm 10,40 \mathrm{~kg}$; talla $157,68 \pm 7,00 \mathrm{~cm}$; edad 13,65 \pm 0,4 años) se dividieron en dos grupos ( 1 experimental y 1 grupo control), el grupo experimental realizó un programa de estiramientos de 7 minutos de duración en las clases de educación física, durante un curso escolar completo ( 9 meses), dos sesiones por semana (31 semanas, 62 sesiones de educación física), con una duración del estiramiento de 15 segundos, utilizando la técnica activa dentro del calentamiento y la técnica pasiva en la vuelta a la calma.

De igual forma, es necesario resaltar la importancia del concepto de iniciación y formación deportiva, que aparece con gran relevancia, según los autores (Giménez, F., Robles, A., Rodríguez, R., 2002), quienes plantean unas edades en específico, además, le agregan el hecho que la iniciación y formación deportiva debe tener en cuenta los procesos y las etapas, que se deben respetar.

\section{OBJETIVO GENERAL}

- Determinar el nivel de flexibilidad en los deportistas en formación del Instituto Municipal de Recreación y de Deporte de Tocancipá, a partir de las características de género, edad y modalidad deportiva.

\section{OBJETIVOS ESPECÍFICOS}

- Medir el nivel de flexibilidad a través del test de Sit and Reach, en la población objeto de estudio.

- Relacionar el comportamiento de los datos de acuerdo con las variables objeto de estudio.

- Presentar los resultados a través de percentiles según el comportamiento por sexo y modalidad deportiva.

- La población estudio que se intervino para la investigación, contó con 334 deportistas con una participación del 87 niñas y 247 niños, de la población total objeto de estudio, en 13 modalidades; baloncesto, capoeira, ciclismo, ciclo montañismo, fútbol, futsal, gimnasia, hockey, porras, taekwondo, tenis de campo, tenis de mesa, voleibol entre las edades de 8 a 17.

\section{HIPÓTESIS}

Para formular la hipótesis de la investigación se establecen las siguientes premisas:

Hipótesis alternativa: Las niñas presentan un rango de amplitud diferente frente a los niños en los isquiotibiales y en la zona lumbar.

Hipótesis nula: Los niños presentan un rango de amplitud igual al de las niñas en los isquiotibiales y en la zona lumbar. 


\begin{tabular}{|c|c|c|c|}
\hline Material & Posición Inicial & Posición Final & $\begin{array}{c}\text { Registro } \\
\text { de } \\
\text { Dato }\end{array}$ \\
\hline $\begin{array}{l}\text { Unidad de } \\
\text { medida; } \\
\text { Centímetros } \\
\text { (cm). }\end{array}$ & $\begin{array}{l}\text { Sentados enfrente del cajón con los pies } \\
\text { apoyados, las piernas completamente } \\
\text { extendidas, con una mano sobre la otra, } \\
\text { extender los brazos por encima del } \\
\text { cajón, realizando una flexión profunda de } \\
\text { tronco y llevando las manos lo más lejos } \\
\text { posible con un movimiento lento y sin } \\
\text { rebotes (Sanz, l., 2002). }\end{array}$ & $\begin{array}{l}\text { El evaluador se va a ubicar al lado, } \\
\text { observando que la parte de la } \\
\text { mano más cerca al sujeto será el } \\
\text { dato que se tomará, obteniendo un } \\
\text { mejor control de los resultados, no } \\
\text { se acepta flexionar las rodillas } \\
\text { durante los tres segundos que } \\
\text { dura realizando la flexión (Sanz, l., } \\
\text { 2002). }\end{array}$ & $\begin{array}{l}\text { La medida se toma } \\
\text { desde la parte más } \\
\text { lejana, en la que el } \\
\text { evaluado } \\
\text { mantenga los tres } \\
\text { segundos. }\end{array}$ \\
\hline
\end{tabular}

\section{VARIABLES DE ESTUDIO}

\begin{tabular}{|c|c|c|c|c|}
\hline Variable & Denominación & $\begin{array}{ll}\text { Tipo } & \text { de } \\
\text { variable } & \end{array}$ & Característica & $\begin{array}{l}\text { Unidad De } \\
\text { Medida }\end{array}$ \\
\hline Sexo: & $\begin{array}{l}\text { Es el conjunto de características físicas, } \\
\text { biológicas, anatómicas y fisiológicas de los } \\
\text { seres humanos, que los definen como hombre } \\
\text { o mujer (OMS, 2015). }\end{array}$ & Independiente & Cualitativo & Niños / Niñas. \\
\hline Edad: & $\begin{array}{l}\text { Tiempo que transcurrido desde el nacimiento de } \\
\text { un ser vivo (Porto, 2009). }\end{array}$ & Independiente & Cuantitativo & $\begin{array}{l}\text { Años } \\
\text { cumplidos. }\end{array}$ \\
\hline Deporte: & $\begin{array}{l}\text { Toda forma de actividad física que a través de } \\
\text { una participación organizada o no, tenga por } \\
\text { objeto la mejora de la condición física y } \\
\text { psíquica, el desarrollo de las relaciones sociales } \\
\text { o la obtención de resultados en competiciones } \\
\text { de cualquier nivel (Carta Europea del Deporte, } \\
\text { 1992). }\end{array}$ & Independiente & Cualitativo & $\begin{array}{l}\text { Combate, } \\
\text { Pelota, } \\
\text { Tiempo y y } \\
\text { marca, Arte y } \\
\text { precisión. }\end{array}$ \\
\hline $\begin{array}{l}\text { Flexibilidad } \\
\text { (Sit and } \\
\text { Reach): }\end{array}$ & $\begin{array}{l}\text { ACSM en } 1995 \text { define la flexibilidad como la } \\
\text { capacidad condicional necesaria para la } \\
\text { correcta ejecución de los movimientos humanos } \\
\text { y deportivos. }\end{array}$ & Dependiente & Cuantitativo & $\begin{array}{l}\text { Centímetros } \\
\text { (cm). }\end{array}$ \\
\hline
\end{tabular}




\section{RESULTADOS}

Se incluyeron 334 deportistas con una participación del 87 niñas y 247 niños, de la población total objeto de estudio, en 13 modalidades; baloncesto, capoeira, ciclismo, ciclo montañismo, fútbol, futsal, gimnasia, hockey, porras, taekwondo, tenis de campo, tenis de mesa, voleibol entre las edades de 8 a 17.

\section{Distribución de la variable sexo y edad}

En la gráfica se observa, el número de los participantes a los diferentes años según el sexo, evidenciando una mayor participación entre los 10 a 14 años en ambos sexos, y un descenso significativo en el número de participantes a los 8 años, donde solo participaron niños de 17 años.

\begin{tabular}{|c|c|c|c|}
\hline Años & Niñas & Niños & Total \\
\hline $\mathbf{8}$ & 0 & 3 & 3 \\
\hline $\mathbf{9}$ & 1 & 11 & 12 \\
\hline $\mathbf{1 0}$ & 5 & 26 & 31 \\
\hline $\mathbf{1 1}$ & 16 & 47 & 63 \\
\hline $\mathbf{1 2}$ & 12 & 62 & 74 \\
\hline $\mathbf{1 3}$ & 20 & 43 & 63 \\
\hline $\mathbf{1 4}$ & 14 & 22 & 36 \\
\hline $\mathbf{1 5}$ & 9 & 11 & 20 \\
\hline $\mathbf{1 6}$ & 9 & 14 & 23 \\
\hline $\mathbf{1 7}$ & 1 & 8 & 9 \\
\hline Total & $\mathbf{8 7}$ & $\mathbf{2 4 7}$ & $\mathbf{3 3 4}$ \\
\hline
\end{tabular}

Distribución de las variables sexo y edad

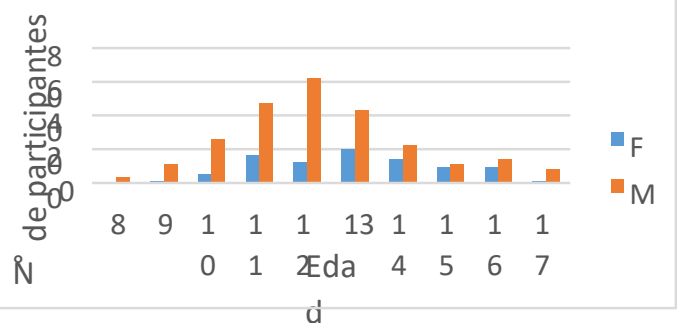




\section{Distribución de la variable Sit and Reach por edad-sexo}

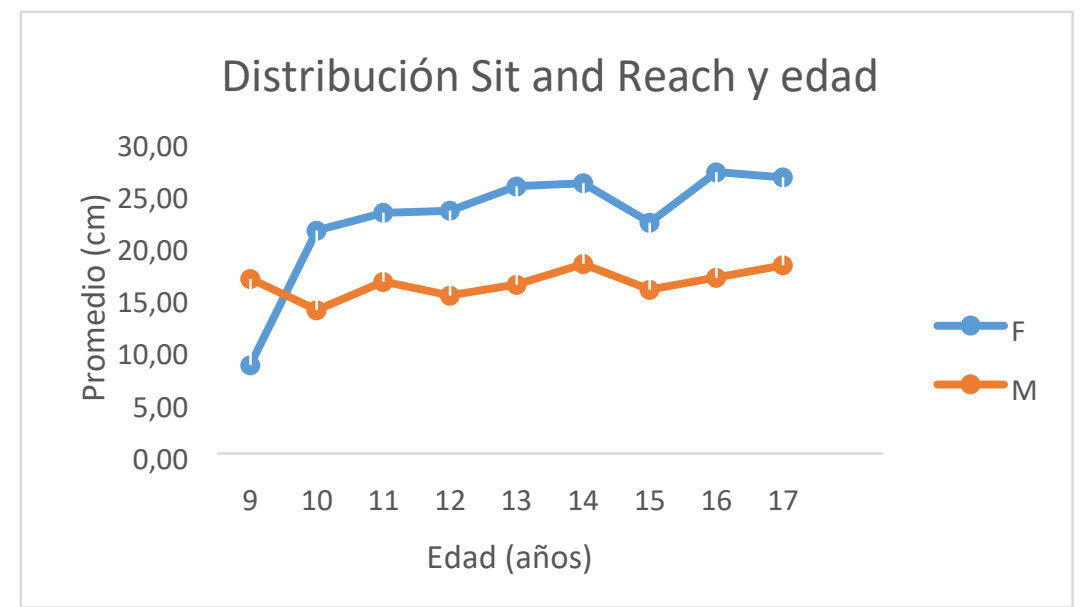

Fuente: Elaboración propia a partir de los hallazgos de la investigación.

\section{Percentiles en niños de acuerdo a la edad y test Sit and Reach}

\begin{tabular}{|c|c|c|c|c|c|c|c|c|c|c|c|c|c|}
\hline EDAD & $\mathbf{1 0} \%$ & $\mathbf{2 0} \%$ & $\mathbf{3 0} \%$ & $\mathbf{4 0} \%$ & $\mathbf{5 0} \%$ & $\mathbf{6 0} \%$ & $\mathbf{7 0} \%$ & $\mathbf{8 0} \%$ & $\mathbf{9 0} \%$ & $\mathbf{N}$ & $\mathbf{X}$ & $\mathbf{D E}$ & Promedio \\
\hline 8 & 17,0 & 18,0 & 19,0 & 20,0 & 21,0 & 22,0 & 22,0 & 22,0 & 22,5 & 6,0 & 20,20 & 2,7 & $20,2 \pm 2,7$ \\
\hline 9 & 11,1 & 13,2 & 14,3 & 15,4 & 17,0 & 17,6 & 18,7 & 19,8 & 22,8 & 22,0 & 16,80 & 4,5 & $16,8 \pm 4,5$ \\
\hline 10 & 6,0 & 11,0 & 13,0 & 15,0 & 16,0 & 17,0 & 17,0 & 18,8 & 20,0 & 52,0 & 14,00 & 5,4 & $14 \pm 5,4$ \\
\hline 11 & 8,0 & 11,0 & 13,0 & 15,5 & 16,8 & 19,4 & 20,0 & 23,2 & 24,0 & 94,0 & 16,50 & 6,6 & $16,5 \pm 6,6$ \\
\hline 12 & 7,7 & 10,0 & 12,0 & 13,0 & 15,0 & 17,0 & 18,5 & 20,0 & 23,0 & 124,0 & 15,20 & 5,7 & $15,2 \pm 5,7$ \\
\hline 13 & 9,0 & 10,5 & 13,5 & 15,0 & 17,0 & 18,0 & 19,5 & 21,0 & 24,3 & 86,0 & 16,20 & 5,9 & $16,2 \pm 5,9$ \\
\hline 14 & 9,0 & 10,6 & 14,0 & 15,2 & 18,0 & 19,0 & 22,1 & 26,0 & 28,0 & 44,0 & 19,30 & 7,6 & $19,3 \pm 7,6$ \\
\hline 15 & 9,0 & 9,4 & 13,6 & 16,0 & 17,0 & 18,6 & 19,7 & 20,0 & 20,0 & 22,0 & 15,70 & 5 & $15,7 \pm 5$ \\
\hline 16 & 3,0 & 6,8 & 10,0 & 12,0 & 19,5 & 22,4 & 24,0 & 26,6 & 28,0 & 28,0 & 16,90 & 9,7 & $16,9 \pm 9,7$ \\
\hline 17 & 7,5 & 15,0 & 16,5 & 17,0 & 18,5 & 20,0 & 20,5 & 24,0 & 27,0 & 16,0 & 18,10 & 7,3 & $18,1 \pm 7,3$ \\
\hline
\end{tabular}

Fuente: Elaboración propia a partir de los hallazgos de la investigación. 
Percentiles en niñas de acuerdo a la edad y test Sit and Reach

\begin{tabular}{|c|c|c|c|c|c|c|c|c|c|c|c|c|c|}
\hline EDAD & $10 \%$ & $20 \%$ & $30 \%$ & $40 \%$ & $50 \%$ & $60 \%$ & $70 \%$ & $80 \%$ & $90 \%$ & $\mathrm{~N}$ & $\dot{X}$ & DE & Promedio \\
\hline 9 & 6 & 6 & 7 & 8 & 9 & 9 & 10 & 11 & 11 & 2 & 8,5 & 4,9 & $8,5 \pm 4,9$ \\
\hline 10 & 16 & 17 & 18 & 20 & 22 & 23 & 25 & 26 & 28 & 10 & 21,4 & 5,8 & $21,4 \pm 5,8$ \\
\hline 11 & 14 & 18 & 21 & 22 & 24 & 25 & 26 & 28 & 29 & 32 & 23,1 & 6,5 & $23,1 \pm 6,5$ \\
\hline 12 & 12 & 18 & 19 & 21 & 24 & 25 & 25 & 29 & 34 & 24 & 23,3 & 8,2 & $23,3 \pm 8,2$ \\
\hline 13 & 19 & 22 & 24 & 25 & 26 & 27 & 28 & 29 & 32 & 40 & 25,6 & 5,3 & $25,6 \pm 5,3$ \\
\hline 14 & 17 & 19 & 23 & 27 & 28 & 29 & 29 & 31 & 33 & 28 & 25,9 & 6,4 & $25,9 \pm 6,4$ \\
\hline 15 & 14 & 16 & 17 & 19 & 21 & 22 & 25 & 28 & 33 & 18 & 22,2 & 7,8 & $22,2 \pm 7,8$ \\
\hline 16 & 18 & 20 & 23 & 27 & 28 & 29 & 31 & 32 & 33 & 18 & 27 & 6,7 & $27 \pm 6,7$ \\
\hline 17 & 26 & 26 & 26 & 26 & 27 & 27 & 27 & 27 & 27 & 2 & 26,5 & 0,7 & $26,5 \pm 0,7$ \\
\hline
\end{tabular}

Fuente: Elaboración propia a partir de los hallazgos de la investigación.

Por medio del test Sit and Reach en la relación edad y género se observa el punto más bajo de flexibilidad $8,5 \mathrm{~cm}$ a los 9 años en las niñas, a los 10 años el comportamiento en niños y niñas varía a favor de las niñas con un valor de $21 \mathrm{~cm}$, desde esta edad la gráfica actúa similar hasta los 14 años, con valor en las niñas de $25 \mathrm{~cm}$., aproximadamente y en los niños $18 \mathrm{~cm}$. , a los 15 años ambos sexos disminuyen el nivel de flexibilidad en $2 \mathrm{~cm}$., con respecto al año anterior, se encuentra el valor máximo en las niñas a los 16 años con $27 \mathrm{~cm}$. , y en niños a los 17 con $18 \mathrm{~cm}$., esto es igual, a que en esta edad se reporta el mayor grado de flexibilidad.

\section{Distribución de la variable Sit and Reach con relación al deporte en niños}

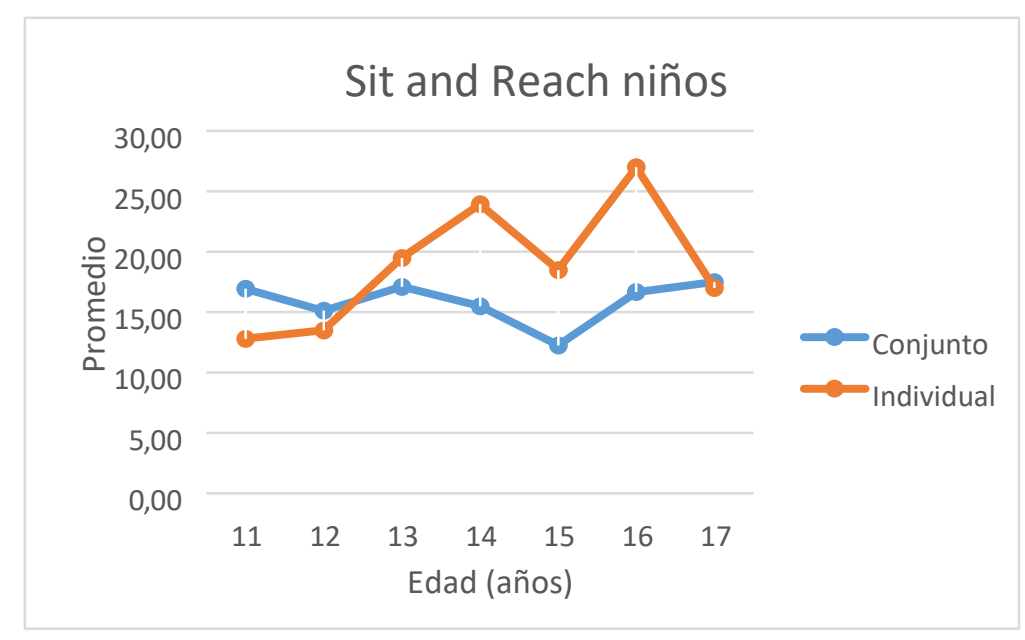

Fuente: Elaboración propia a partir de los hallazgos de la investigación. 
Percentiles en niños de acuerdo al deporte y test Sit and Reach

\begin{tabular}{|l|l|l|l|l|l|l|l|l|l|l|l|l|l|}
\hline DEPORTE & $\mathbf{1 0} \%$ & $\mathbf{2 0} \%$ & $\mathbf{3 0} \%$ & $\mathbf{4 0} \%$ & $\mathbf{5 0} \%$ & $\mathbf{6 0} \%$ & $\mathbf{7 0} \%$ & $\mathbf{8 0} \%$ & $\mathbf{9 0 \%}$ & $\mathbf{N}$ & $\mathbf{x}$ & DE & Promedio \\
\hline BALONCESTO: & 22 & 22 & 22 & 22 & 23 & 23 & 23 & 23 & 23 & 2 & 22,5 & 0,7 & $22,5 \pm 0,7$ \\
\hline CAPOEIRA: & 14 & 22 & 23 & 23 & 25 & 25 & 26 & 32 & 33 & 14 & 24,5 & 7,1 & $24,5 \pm 7,1$ \\
\hline CICLISMO: & 16 & 17 & 19 & 20 & 22 & 23 & 25 & 26 & 28 & 4 & 21,8 & 6,1 & $21,8 \pm 6,1$ \\
\hline CICLO MONTAÑISMO: & 15 & 15 & 16 & 18 & 19 & 20 & 22 & 24 & 26 & 10 & 19,6 & 4,7 & $19,6 \pm 4,7$ \\
\hline FÚTBOL: & 5 & 10 & 13 & 15 & 16 & 17 & 19 & 21 & 24 & 308 & 15,2 & 7 & $15,2 \pm 7$ \\
\hline FUTSAL: & 4 & 7 & 9 & 11 & 13 & 15 & 17 & 20 & 21 & 68 & 13,3 & 6,8 & $13,3 \pm 6,8$ \\
\hline GIMNASIA: & 26 & 26 & 27 & 27 & 27 & 27 & 27 & 28 & 28 & 2 & 27 & 1,4 & $27 \pm 1,4$ \\
\hline HOCKEY: & 7 & 9 & 11 & 12 & 14 & 15 & 16 & 18 & 20 & 8 & 13,4 & 5,4 & $13,4 \pm 5,4$ \\
\hline TAEKWONDO: & 10 & 11 & 13 & 16 & 18 & 18 & 20 & 24 & 25 & 14 & 17,4 & 6,6 & $17,4 \pm 6,6$ \\
\hline TENIS DE CAMPO: & 5 & 5 & 11 & 17 & 19 & 20 & 24 & 27 & 28 & 6 & 16,8 & 10,4 & $16,8 \pm 10,4$ \\
\hline TENIS DE MESA: & 8 & 9 & 10 & 11 & 11 & 11 & 12 & 12 & 15 & 6 & 11 & 3,6 & $11 \pm 3,6$ \\
\hline VOLEIBOL: & 10 & 11 & 13 & 16 & 18 & 18 & 20 & 24 & 25 & 6 & 16,3 & 5,8 & $16,3 \pm 5,8$ \\
\hline
\end{tabular}

Fuente: Elaboración propia a partir de los hallazgos de la investigación.

Los niños en deportes individuales presentan el menor valor de flexibilidad a los 11 años con $12 \mathrm{~cm}$., mientras que los que practican deporte en conjunto presentan el menor valor a los 15 años con $12 \mathrm{~cm}$., a los 16 años con $27 \mathrm{~cm}$., se observa el mayor grado de flexibilidad.
Relacionando las variables objeto de estudio, los valores porcentuales que se manejaron se muestran en las tablas de percentiles que fueron clasificadas por sexo, edad y deporte, presentando los valores de los percentiles $10 \%$ al $90 \%$, que se pueden observar en los anexos 1 al 8.

\section{Distribución de la variable Sit and Reach con relación al deporte en niñas}

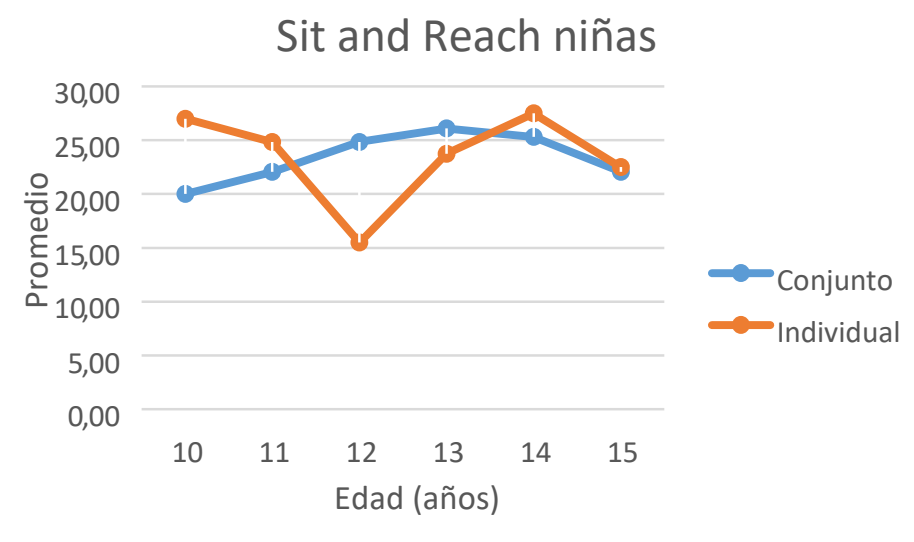

Fuente: Elaboración propia a partir de los hallazgos de la investigación. 


\section{Percentiles en niñas de acuerdo al deporte y test Sit and Reach}

\begin{tabular}{|l|l|l|l|l|l|l|l|l|l|l|l|l|l|}
\hline DEPORTE & $\mathbf{1 0} \%$ & $\mathbf{2 0} \%$ & $\mathbf{3 0} \%$ & $\mathbf{4 0} \%$ & $\mathbf{5 0} \%$ & $\mathbf{6 0} \%$ & $\mathbf{7 0} \%$ & $\mathbf{8 0} \%$ & $\mathbf{9 0} \%$ & $\mathbf{N}$ & $\mathbf{X}$ & DE & Promedio \\
\hline BALONCESTO: & 17 & 18 & 18 & 19 & $\mathbf{1 9}$ & $\mathbf{1 9}$ & 20 & 20 & 21 & 2 & 19 & 2,8 & $19 \pm 2,8$ \\
\hline CAPOEIRA: & 25 & 26 & 28 & 29 & 29 & 29 & 30 & 31 & 36 & 12 & 29,5 & 3,8 & $29,5 \pm 3,8$ \\
\hline FÚTBOL: & 10 & 12 & 13 & 15 & 21 & 25 & 25 & 27 & 29 & 8 & 19,6 & 9,4 & $19,6 \pm 9,4$ \\
\hline FUTSAL: & 32 & 33 & 33 & 33 & 34 & 34 & 34 & 34 & 35 & 2 & 33,5 & 2,1 & $33,5 \pm 2,1$ \\
\hline GIMNASIA: & 22 & 23 & 24 & 24 & 25 & 25 & 25 & 27 & 31 & 10 & 25,4 & 4,3 & $25,4 \pm 4,3$ \\
\hline HOCKEY: & 32 & 32 & 33 & 33 & 33 & 33 & 33 & 34 & 34 & 2 & 33 & 1,4 & $33 \pm 1,4$ \\
\hline PORRAS: & 19 & 22 & 24 & 26 & 27 & 28 & 29 & 32 & 34 & 48 & 26,6 & 6,2 & $26,6 \pm 6,2$ \\
\hline TAEKWONDO: & 20 & 21 & 22 & 22 & 23 & 24 & 24 & 24 & 25 & 4 & 22,5 & 2,6 & $22,5 \pm 2,6$ \\
\hline TENIS DE CAMPO: & 19 & 19 & 20 & 21 & 22 & 22 & 23 & 24 & 24 & 2 & 21,5 & 4,9 & $21,5 \pm 4,9$ \\
\hline TENIS DE MESA: & 12 & 13 & 14 & 15 & 17 & 18 & 19 & 20 & 24 & 10 & 17,3 & 5,1 & $17,3 \pm 5,1$ \\
\hline VOLEIBOL: & 14 & 17 & 20 & 21 & 23 & 25 & 27 & 28 & 31 & 74 & 23 & 6,9 & $23 \pm 6,9$ \\
\hline
\end{tabular}

Fuente: Elaboración propia a partir de los hallazgos de la investigación.

La gráfica muestra el análisis de la variable, que examina la asociación entre deportes de conjunto (baloncesto, futsal, hockey, porras y voleibol), e individuales (capoeira, ciclismo, cliclomontañismo, gimnasia, taekwondo, tenis de campo y tenis de mesa) con la variable sexo mediante la prueba Sit and Reach para toda la muestra, así como para niños y niñas por separado.

De acuerdo con la gráfica, se observa que las niñas que practican deportes individuales tienen un nivel de flexibilidad mayor a los 10 años con $27 \mathrm{~cm}$., mientras que entre los $11 \mathrm{y}$ 13 años predomina la flexibilidad en los deportes de conjunto. A los 14 años las niñas presentan un mayor rango de flexibilidad con un valor de $28 \mathrm{~cm}$.

\section{DISCUSIÓN}

A partir de los percentiles que describen el comportamiento de la variable de flexibilidad obtenidos con el test de Sit and Reach en niños de formación del Instituto
Municipal de Recreación y de Deporte de Tocancipá, se expone los siguientes postulados:

Con relación a los resultados expuestos se identificaron los percentiles respectivos para cada variable, en un estudio realizado por Silvio Villera Coronado (2010) en niños escolares de 10, 11 y 12 años, los valores obtenidos muestran que los niveles en las niñas evaluadas en el percentil 90 reporta $36,8 \mathrm{~cm} ., 40,5 \mathrm{~cm} ., 29 \mathrm{~cm}$., respectivamente, en los niños el registro fue $36 \mathrm{~cm}$., $36,5 \mathrm{~cm}$., $39,1 \mathrm{~cm}$. , mientras los niños del Instituto Municipal de Recreación y de Deporte de Tocancipá, presentaron un valor bajo en comparación con el estudio nombrado anteriormente, con un percentil 90 para niñas de $28 \mathrm{~cm} ., 29 \mathrm{~cm}$. y $34 \mathrm{~cm}$., para niños $20 \mathrm{~cm}$., $24 \mathrm{~cm}$. y $23 \mathrm{~cm}$. en los años de 10, 11 y 12.

De la misma manera, un estudio de Bustamante y colaboradores (2012) se establecieron percentiles para niños de 7 a 17 años, donde se registrando un percentil 90 para los años de 9 a 17 de la siguiente 
manera, en niños a los 9 años $26 \mathrm{~cm} ., 10$ años $25 \mathrm{~cm}$., 11 años $24 \mathrm{~cm}, 12$ años $23 \mathrm{~cm}$., 13 años $24 \mathrm{~cm} .14$ años $25 \mathrm{~cm}$., 15 años 26 cm., 16 años $27 \mathrm{~cm}$., 17 años $28 \mathrm{~cm}$., y en niñas $27 \mathrm{~cm} ., 27 \mathrm{~cm} ., 27 \mathrm{~cm} ., 27 \mathrm{~cm} ., 27 \mathrm{~cm}$., $28 \mathrm{~cm} ., 29 \mathrm{~cm} ., 30 \mathrm{~cm}$., respectivamente, en relación a lo anterior, los percentiles de los niños en formación de Tocancipá, se encuentran por debajo según los valores de este estudio.

Otros estudios relacionados con la edad muestran que las niñas son más flexibles que los niños en las edades de 13 a 16 años según, Maffuli, N., Cols. (1994). Encuentran que la mayoría de los rangos de movimiento son influenciados a través de la edad y que algunos son específicos de cada deporte. La aparición de la pubertad tiene mayor valor predictivo que la edad cronológica, se evidencia una disminución relativa en flexibilidad durante el periodo de crecimiento lineal máximo, de acuerdo con los datos obtenidos durante el periodo investigativo, se evidenció que las niñas en la misma edad tienen un mayor rango de flexibilidad, mientras que, los niños mantienen picos en el resultado de la variable.

La flexibilidad en el deporte se manifiesta como parte integral de un programa de entrenamiento físico, con el fin de disminuir el riesgo de lesiones, aliviar el dolor muscular post ejercicio y mejorar el rendimiento deportivo, para determinar el nivel de flexibilidad en cada deporte se establecieron percentiles, donde se observaron los resultados según la variable relacionada al deporte, Álvarez, J., (2003) plantea: que los niños en categoría de formación en fútbol presentan valores de 18 a $25 \mathrm{~cm}$, en comparación con los niños de Tocancipá que practican el mismo deporte y se manifiesta un promedio de $(19,6 \pm 9,4 \mathrm{~cm})$ para niñas, $(15,2 \pm 7 \mathrm{~cm})$ para niños.

Estudios como este permiten dar una noción de las características de la amplitud articular de un grupo de deportistas, en el municipio de Tocancipá de acuerdo con la modalidad deportiva se observó que el deporte Capoeira fue el que presentó un mayor rango de movimiento en el test de Sit and Reach. (Yannick, L., 2010) expone que la flexibilidad es una capacidad básica de gran importancia en Capoeira, debido a que permite una amplitud de recorrido de las articulaciones, de los diversos músculos.

La flexibilidad se puede ganar y mejorar a través de esta práctica deportiva, porque proporciona una mayor agilidad y destreza mejorando el rendimiento deportivo.

Desde el punto de vista comparativo entre niñas y niños del Instituto Municipal de Recreación y de Deporte de Tocancipá, se observa que las niñas presentan un mayor rango de flexibilidad según el test Sit and Reach, que desde los 12 años $(23,3 \pm 8,2 \mathrm{~cm})$ a los 14 años $(25,9 \pm 6,4 \mathrm{~cm})$ se muestra un aumento significativo en este estudio.

\section{CONCLUSIÓN}

Se concluye que, para la valoración del test físico, los niños y niñas del Instituto Municipal de Recreación y de Deporte de Tocancipá, se logra obtener los resultados de los mejores promedios del test Sit and Reach en niñas, el cual se presenta a los 14 años con un valor de $(25,9 \pm 6,4 \mathrm{~cm})$ y en los niños de $(19,3 \pm 7,6 \mathrm{~cm})$, a partir de los datos obtenidos se observó que el nivel de flexibilidad en niñas es más alto con respecto al de los niños. A partir de las variables objeto de estudio que se establecieron (edad, deporte y sexo), se observó un predominio de 
flexibilidad en niñas, al presentar un mayor nivel en el test Sit and Reach; de este modo capoeira en niñas obtuvo un valor de $(29,5 \pm 3,8 \mathrm{~cm})$ y en niños $(24,5 \pm 7,1 \mathrm{~cm})$ es el deporte que tiene un mejor resultado en el test Sit and Reach en ambos sexos.

Teniendo en cuenta, los resultados de la prueba de hipótesis donde se comparan los promedios de sexo, con la variable flexibilidad a través de la prueba T-student, se confirma hipótesis alternativa, las niñas presentan un rango de amplitud diferente frente a los niños en los isquiotibiales y en la zona lumbar ( $p: 0,0087$ ).

Por otra parte, la investigación realizada con los niños y niñas del Instituto Municipal de Recreación y de Deporte de Tocancipá, permitió reconocer el percentil 90, de acuerdo a la edad, las niñas muestran un rango entre 22,5 a $27 \mathrm{~cm}$., y en los niños 11 $\mathrm{cm}$ a $27 \mathrm{~cm}$, a su vez, la variable Sit and Reach y deporte presentan un percentil 90 de 21 a $31 \mathrm{~cm}$., en niñas y en niños de 23 a $25 \mathrm{~cm}$., teniendo en cuenta, lo anterior 46 niños y 41 niñas dependiendo el rango de cada edad se encuentran arriba del percentil 90, de esta manera, se puede determinar que están en proyección para ser talentos deportivos.

Finalmente, se observó el promedio del percentil 20, donde 19 niñas y 46 niños se encuentran por debajo del rango de su edad, estando en zona de riesgo para presentar lesiones, posteriormente, perder el rango de movilidad de las articulaciones.

\section{REFERENCIAS BIBLIOGRÁFICAS}

ACSM. (2010). Las pautas de ACSM (Colegio Americano Estadounidense de Medicna del Deporte) para pruebas de ejercicio y prescripción. $8^{\mathrm{a}}$ ed. Filadelfia, Pensilvania: Lippincott, Williams y Wilkins.

Allen, W., Jackson y Baker, A. (1986). The relationship of the sit and reach test to criterion measures of hamstring and back flexibility in young females. Research quarterly for exercise and sport. USA, v. 57, n 3, pp. 183-186

Alter, M. (1998). Los estiramientos. Bases científicas y desarrollo de ejercicios. Barcelona: Editorial Paidotribo.

Álvarez, J., Casajús, J., \& Corona, P. (2003). Práctica del fútbol, evolución de parámetros cineantropométricos y diferentes aspectos de la condición física en las edades escolares. Apunts. Educación Física y Deportes (72), pp. 28-34.

American College of Sports Medicine (2011). Fundamentos para hábitos de vida saludable.

Arregui, E. y Martínez de Haro, V. (2001). Estado actual de las investigaciones sobre la flexibilidad en la adolescencia. Revista Internacional de Medicina y Ciencias de la Actividad Física y el Deporte vol. 1 (2), pp. 127-135.

Ayala, F., Sainz de Baranda, B., Croixc, S. y Santonja, D. (2012). Fiabilidad y validez de las pruebas sit-and-reach: revisión sistemática. Revista Andaluza Medicina del Deporte.5(2):5362.

Ayala, F., Sainz de Baranda, M. (2012). De Ste Croix y F. Santonja. Rev Andal Med Deporte. 5(2):57-66 
Baumgartner, T., \& Jackson, A. (1995). Measuremat fw Eoaluation in physical educalion and exercise sciozce. Dubuque, IA: Brown \& Benchmark.

Benavent, J., Tella, V., González, I., Colado, J. (2008). Comparación de diferentes tests de campo para la evaluación de la flexibilidad general activa. Fit Perf J. 7(1):26-9.

Blanco, A., ("s.f"). Bases teóricas la preparación física en la edad escolar inicial, Editorial paidotribo, pp. 25, 26,27.

Bustamante, A., Beunen, J., Maia, L. (2012). Valoración de la aptitud física en niños y adolescentes: construcción de cartas percentílicas para la región central del perú, Rev. Perú Med Exp Salud Pública. 29(2):188-97.

Código de la Infancia y la Adolescencia. (2006). Artículo 3: "Para todos los efectos de esta ley son sujetos titulares de derechos todas las personas menores de 18 años. Sin perjuicio de lo establecido en el artículo 34 del Código Civil, se entiende por niño o niña las personas entre los 0 y los 12 años, y por adolescente, las personas entre 12 y 18 años de edad".

Código de Núremberg Tribunal Internacional de Núremberg (1947). Apartado 1.

Cuberek, R., Machová, I., Lipenská, M. (2013). Reliability of v sit-and-reach test used for flexibility self-assessment in females, Acta Univ. Palacki. Olomuc., Gymn. vol. 43, no. 1.

De Castro, G., Suárez, L., Gutiérrez, J. (2012). VIII Seminario Nacional de Nutrición, Medicina y Rendimiento Deportivo. Congreso Internacional de Ciencias del Deporte y la Educación Física. (Sevilla), España: Universidad Pablo de Olavide. Pontevedra, España, pp.10-12.

De Souza Bezerra y colaboradores (2015). Influência da modificação do teste de sentar e alcançar sobre o indicador de flexibilidade em diferentes faixas etárias, vol. 11, n. 3, pp. 310.

Docherty, D., Bell, R. (1985). The relationship between flexibility and linearity measures in boys and girls 6-15 years of age. Journal of human movement studies (Edinburgh, Eng.) 11(5), pp. 279-288.

Farrally, M. (1982). The physical fitness of Scottish schoolboys aged 13 and 17 years. Evaluation of motor fitness. Belgium, Council of Europe committee for development of Sport, pp. 93-108.

Freitas, D., Maia, J., Beunen, G., Lefevre, J., Claessens, A., Marques, A., et al. (2002). Crescimento somático, maturação biológica, aptidão física, actividade física e estatuto socioeconómico de crianças e adolescentes madeirenses. O Estudo de Crescimento da Madeira. Funchal: Universidade da Madeira.

Giménez, F., Robles, M., Robles, J. (2010). El proceso de formación del jugador durante la etapa de iniciación deportiva. Universidad de Huelva, pp. 47-55.

González, I. (1997-98). Validación de pruebas de campo para la medición de la flexibilidad y su relación con la estructura corporal. Tesis doctoral. Facultad de Biología. Sevilla: Universidad de León.

Grosser, M. y Múller, H. (1992). Desarrollo muscular. Un nuevo concepto de musculación. (Power-stretch). Barcelona: Hispano-europea.

Hernández, R., Fernández, C., Baptista, P. (2006). Metodología de la investigación. México: Editorias Mc. Graw Hill, p.216

Heyward, V. (1996). Evaluación y prescripción del ejercicio. Barcelona: Editorial Paidotribo. 
Ley 1306 (2009). Artículo 53: "Para todos los efectos legales el impúber se equipara al niño y niña definido en el artículo 3 del Código de la Infancia y la Adolescencia. De igual manera, el menor adulto se equipara al adolescente de ese estatuto-".

Litwin, J. y Fernández, G. (1984). Evaluación y estadísticas aplicadas a la educación física y el deporte. Buenos Aires: Stadium.

Maffulli, N., king, J., Helms, P. (1994). Training in elite young athletes (the Training of Young Athletes (TOYA) Study): injuries, flexibility and isometric strength, British journal of sports medicine (Guildford, England) 28(2), June 123-136.

Moras, G., Torres, S. (1989). El flexómetro: nuevo test para medir la flexibilidad, Revista de Entrenamiento Deportivo, v. III, n. 3, pp. 14-20.

Norris, C. (1996). La flexibilidad: Principios y práctica. Barcelona: Editorial Paidotribo.

OMS. (2013). Asamblea mundial de la salud- OMS. (2015). Nota descriptiva. № 403.

Peralta, H. (2006). Centros de educación física, escuelas de iniciación y formación deportiva. Una alternativa en cultura física para Colombia, a través de experiencias en el departamento de Cundinamarca. Bogotá, D.C.: Ed. Antropos, pp.17-24.

Ruíz, J. (2003). La evaluación de las capacidades físicas. Técnicas, instrumentos y registro de los datos. Las pruebas de capacidad física: Usos y valor formativo. Cuerpo de Profesores de Enseñanza Secundaria. España: Editorial Mad, S.R.L.

Sainz, P. (2009) El trabajo de la flexibilidad en educación física: Programa de intervención Cultura, Ciencia y Deporte, vol. 4, núm. 10, febrero, España Universidad Católica San Antonio de Murcia, España, pp. 33-38.

Sainz, P., Ayala, F., Murcia, A. (2007-2010). Descripción y análisis de la utilidad de las pruebas sit-and-reach para la estimación de la flexibilidad de la musculatura isquiosural, PCTRM, Universidad Católica San Antonio de Murcia, pp. 1-15.

Sánchez, E., Águila, M. y Rojas, J. (2001). Consideraciones generales acerca del uso de la flexibilidad en el béisbol. Lecturas Educación Física y deportes, n³6, mayo. Recuperado el 22 de marzo.

Sanz, I. (2002). Natación y flexibilidad. Revista Internacional de Medicina y Ciencias de la Actividad Física y el Deporte, vol. 2 (6), pp. 128-142.

Subiela, J. (1978). Entrenamiento Físico. Caracas, Venezuela: Editado por José Vicente Subiela. Turrado, R., Fraile, H. (2016). Grado de Educación Primaria. Mención Educación Física. Wells, K., Dillon, E. (1952). The sit-and-reach. A test of back and leg flexibility, Res Q.; 23:115-8. Werner, W. (2005). Ejercicio y salud, p 34.

\section{WEBGRAFÍA}

Coronado, S., Soto, J. (2010). Valoración de la aptitud física de los escolares de 10 a 12 años de Montería, Colombia. Revista Digital. Buenos Aires, Año 15, No. 148.

Giménez, F., \& Castillo, E. (2002). El tratamiento de la iniciación deportiva. Efdeportes, 1-3.

Muñoz, D. (2009). Flexibilidad: Evidencia Científica y Metodología del Entrenamiento Revista Digital - Buenos Aires - Año 14 - № 131 - Pontificia Universidad Católica de Valparaíso, Chile. 